\title{
Numerical Solution of MEW Equation with Splitting Technique
}

\author{
Melike Karta
}

\begin{abstract}
In this study MEW equation of form
\end{abstract}
$U_{t}+\epsilon U^{2} U_{x}-\mu U_{x x t}=0$

is splitted into two sub-equations such that one is linear and the other nonlinear by following form

$U_{t-} \mu U_{x x t}=0$

$U_{t}+\epsilon U^{2} U_{x}-\mu U_{x x t}=0$

Sub-equations with initial and boundary conditions are numericallay solved with finite difference method by help linearization technique using strang siplitting techniques. MEW equation are calculated error norms $L_{2}, L_{\alpha}$ and invariants. Calculated values are compared with those available in the literature.

Index Terms - Finite difference method, MEW equation, Splitting technique.

\section{INTRODUCTION}

The one- dimensional generalized equal width (GEW) equation is of the form

$$
U_{t}+\epsilon U^{p} U_{x}-\mu U_{x x t}=0
$$

With the physical boundary conditions $U \rightarrow 0$ as $x \rightarrow{ }_{-}^{+} \infty$, respectively, where $t$ is time $x$ is space coordinate. $p_{s} \in$ and $\mu$ are positive parameters.In equation (1), when $\mathrm{p}=1$ we obtain equal width (EW) equation introduced by Morrison and et al.[10] when $p=2_{x} \in=3$, we obtain modified equal width (MEW) equation of the form

$$
U_{t}+\epsilon U^{2} U_{x}-\mu U_{x x t}=0
$$

The MEW equation with a limited set of boundary and conditions have an analytical solution as the EW equation. Thus,numerical solution of the MEW equation has been calculated by many author. Esen and Kutluay [1] used a linearized numerical scheme based on finite difference method to obtain solitary wave solutains of one- dimensional generalized equal width (MEW) equation.Evans and R.Raslan [2] studied the generalized GEW equation by using collocation method with help quadratic B-splines. .Hamdi and et al [4].derived exact solitary wave solutions of the (GEW) equation. Karakoç and et al. [5] solved numerically MEW equation by giving two different linearization techniques based on collocation finite element method using cubic b-splines approximate functions.Karakoç and Geyikli [6] a numerical solution of the modified equal width wave (MEW) equation solved with subdomain method using sextic B-spline. Raslan K. R ,A.Ramadan, M, Ameen, I. [7] solved modified equal width (MEW) equation by using the finite diffrence method. Keskin and Irk [8] presented a numerical study of the modified equal width (MEW) equation by using Letter, Ağrı İbrahim Çeçen University, Ağrı, Turkey the finite diffrence method. Saka [12] proposed quntic b-spline collocation algorithms for numerical solution of the modified equal width (MEW) equation.Wazwaz [16] investigated the MEW equation and two of its variants by asine-cosine ansatz and thetanh method. Zaki [18] used a quintic b- spline collocation method to investigate the motion of asingle solitary wave interaction of two solitary waves and birth of solitons for the MEW equation.

This article is organized as follows: In section 2, strang splitting technique is briefly introduced. In section 3,Equation (2) is split into two sub-equations and sub-equations with initial -boundary conditions (5)-(6) are numerically solved using Crank- Nicolson finite difference approximation and a linearization technique. In section 4 , the error norms $L_{2}$ and $L_{\alpha}$ are calculated and compared with those available in the literatüre. In section 5, a brief conclision is given.

\section{SPLITTING TECHNIQUE}

The second order symmetric [9] or more widely known strang splitting technique [15] is obtained and its scheme is given by

$$
\begin{aligned}
& \frac{d U^{*}(t)}{d t}=A U^{*}(t), U^{*}\left(t_{n}\right)=U_{n}^{0} \quad t \in\left[t_{n}, t_{n+\frac{2}{2}}\right] \\
& \frac{d U^{-*}(t)}{d t}=B U^{* *}(t), U^{* 8}\left(t_{n}\right)=U^{*}\left(t_{n+\frac{1}{2}}\right), \quad t \in\left[t_{n}, t_{n+1}\right]
\end{aligned}
$$

$$
\frac{d U^{m}(t)}{d t}=A U^{* * *}(t), U^{* * *}\left(t_{n+\frac{2}{2}}\right)=U^{* *}\left(t_{n+1}\right), \quad t \in\left[t_{n+\frac{2 n}{2}} t_{n+1}\right]
$$

Where $t_{n+\frac{1}{2}}=t_{n}+\frac{\Delta t}{2}$ and the desired solution is obtained by $U\left(t_{n+1}\right)=U^{* * 4}\left(t_{n+1}\right)$. The splitting error of scheme is [3]

$E=\frac{(\Delta t)^{2}}{24}\left(\left[A_{x}\left[B_{x} A\right]\right]-2\left[B_{x}\left[A_{x} B\right]\right]\right) U\left(t_{n}\right)+O(\Delta t)^{a}$

$\left[A_{x} B\right]=A B-B A$

is defined as the commutator of two operators and the brackets are known as "Lie bracket "in the literatüre [17].Thus, the strang splitting has the second order accuracy.

\section{APPLCATION OF THE METHOD}

In this paper, we have split the one-dimensional modified equal width $\mathrm{MEW}$ equation by the following form

$$
U_{t}+e u^{2} U_{x}-\mu U_{x x t}=0
$$


two sub-equations such that one is linear and the other nonlinear of form

$$
U_{t-1} \mu U_{x x t}=0
$$

and

$$
U_{t}+e u^{2} U_{x}-\mu U_{x x t}=0
$$

with the boundary and initial condition

$$
\begin{aligned}
& U\left(a_{x} t\right)=0_{x} U(b, t)=0 \\
& U\left(x_{x}, 0\right)=A \operatorname{sech}\left(k\left(x-x_{0}\right)\right)
\end{aligned}
$$

using strang splitting techniques by help a linearization technique.

Procedure for Paper Submission

Where $A=\sqrt{\frac{\epsilon(p+1)(p+2)}{2 \epsilon}}$ is amplitude of dipendent solitary wave $v=A_{/} /^{2}$ is velocity of wave, $k=1 / \mu$ is number-wave .Further, $c=1 / 32^{v} p=2, \quad \epsilon=3 x_{0}=30$. Analitical solution of the MEW equation (2)

$U(x, t)=A\left(k\left[x-x_{0}-v t\right]\right)$

Let use assume that the solution domain of the problem given by equation (2) with condition boundary-initial (5) and (6) is bounded by region $a \leq x \leq b$. The interval $[a, b]$ is divided into $N$ equal suninterval such that $a<x_{0}<x_{1}<\ldots<\alpha_{N}=b$ for $m=0_{x} 1_{x a m} N$ at the nodal points $x_{m}$ by selecting the space step size as $h=\frac{b-a}{N}=\left(x_{m+1}-x_{m}\right)$.

Throught paper, we have used the forward difference approximation for $U_{t}, U_{x x t}$, central difference for $U_{x}$ and the Crank-nicolsan difference approximation for $U^{2} U_{x}$ in equation (2) lead to

$\left.\frac{U_{m}^{n+1}-U_{m}^{n}}{\Delta t}+\frac{\epsilon}{2}\left[\left(U^{2} U_{x}^{n+1}\right)+\left(U^{2} U_{x}^{n}\right)\right)\right]=\frac{\mu}{\Delta t}\left[U_{x x}^{n+1}-U_{x x}^{n}\right]$

implementing Rubin and Graves linearization technique [14] to equation (2)

$\left(U^{2} U_{x}\right)^{n+1}=U^{n+1} U^{n} U_{x}^{n}+U^{n} U^{n+1} U_{x}^{n}+U^{n} U^{n} U_{x}^{n+1}-2 U^{n} U^{n} U_{x}^{n}$

We obtain

$\left[\frac{\mu}{\Delta t h^{2}}\right] U_{m-1}^{n+1}+\left[\frac{2 \mu}{\Delta t h^{2}}+\frac{1}{\Delta t}\right] U_{m}^{n+1}+\left[-\frac{\mu}{\Delta t h^{2}}\right] U_{m+1}^{n+1}$

$=\left[-\frac{\mu}{\Delta t h^{2}}\right] U_{m-1}^{n}+\left[\frac{2 \mu}{\Delta t h^{2}}+\frac{1}{\Delta t}\right] U_{m}^{n}+\left[-\frac{\mu}{\Delta t h^{2}}\right] U_{m+1}^{n}$

and

$\left\lfloor-\frac{\epsilon}{4 h}\left(U_{m}^{n}\right)^{2}-\frac{\mu}{\Delta t h^{2}}\right\rfloor U_{m+2}^{n+1}+\left\lfloor\frac{1}{\Delta t}+\frac{\epsilon}{2 h} U_{m}^{n}\left(U_{m+1}^{n}-U_{m-1}^{n}\right)+\frac{2 \mu}{\Delta t h^{2}}\right\rfloor U_{m}^{n+2}+$

$\left\lfloor\frac{\epsilon}{\Delta h}\left(U_{m}^{n}\right)^{2}-\frac{\mu}{\Delta t h^{2}}\right\rfloor U_{m+1}^{n+1}=\left\lfloor-\frac{\mu}{\Delta t h^{2}}\right\rfloor U_{m-1}^{n}+\left\lfloor\frac{2 \mu}{\Delta t h^{2}}+\frac{1}{\Delta t}\right\rfloor U_{m}^{n}++\left[-\frac{\mu}{\Delta t h^{2}}\right] U_{m+2}^{n}$

for $\mathrm{m}=1,2, \ldots, \mathrm{N}$
IV. NUMERICAL EXAMPLES AND RESULTS

The MEW equation (2) has three invariant conditions to be mass, momentum, and energy respectively [11]

$I_{1}=\int_{a}^{b} U d x \bumpeq h \sum_{j=1}^{N}\left(U_{j}^{n}\right)$

$\left.I_{2}=\int_{Q}^{0} U^{2}+\mu\left(U_{x}\right)^{2} d x \cong h \sum_{j=1}^{W}\left(U_{j}^{n}\right)^{2}+\mu\left(U_{x}\right)_{j}^{n}\right)$

$I_{a}=\int_{a}^{b} U^{4} d x \bumpeq h \sum_{j=1}^{N}\left(U_{j}^{n}\right)^{4}$

to show the performence of the method, error norms $L_{2}$ and $L_{\alpha}$ are calculated

$L_{2}=\left\|U^{\text {exact }}-U_{N}\right\|_{2} \cong \sqrt{h \sum_{j=0}^{N}\left|U_{j}^{\text {gxact }}-\left(U_{N}\right)_{j}\right|^{2}}$

\begin{tabular}{|c|c|c|c|c|c|c|}
\hline & \multicolumn{2}{|c|}{ present method } & \multicolumn{2}{|l|}{ Ref.[12] } & \multicolumn{2}{|l|}{ Ref.[5] } \\
\hline $\mathrm{T}$ & $\begin{array}{l}L_{2} \\
10^{3}\end{array}$ & $\begin{array}{l}\mathbb{L}_{m a} \\
10^{3}\end{array}$ & $\begin{array}{l}L_{2} \\
10^{a}\end{array}$ & $\begin{array}{l}L_{m a} X \\
10^{3}\end{array}$ & $L_{2} \times 10^{3}$ & $L_{m=0} \times 10^{2}$ \\
\hline 5 & 0.00089 & 0.00055 & 0.00007 & 0.00008 & 0.0447267 & 0.0423438 \\
\hline 10 & 0.00177 & 0.00111 & 0.00014 & 0.00016 & 0.0890842 & 0.0867198 \\
\hline 15 & 0.00266 & 0.00168 & 0.00021 & 0.00024 & 0.1327126 & 0.1316924 \\
\hline 20 & 0.00354 & 0.00225 & 0.00027 & 0.00032 & 0.1752706 & 0.1764596 \\
\hline
\end{tabular}

$L_{\infty}=\left\|U^{\text {exact }}-U_{N}\right\|_{\infty} \cong \max \left|U^{\text {exact }}-\left(U_{N}\right)_{j}\right|$

all computations have been done using matlab program.

$\mathrm{h}=0.1, \mathrm{k}=0.05, \mathrm{tf}=20,0 \leq \mathrm{x} \leq 80$

$\mathrm{h}=0.1, \mathrm{k}=0.2, \mathrm{tf}=80,0 \leq \mathrm{x} \leq 80$

\begin{tabular}{|l|l|l|l|l|l|l|}
\hline & \multicolumn{3}{|c|}{ present method } & \multicolumn{3}{c|}{ Ref.[1] } \\
\hline $\mathrm{T}$ & $I_{1}$ & $I_{2}$ & $I_{3}$ & $I_{1}$ & $I_{2}$ & $I_{3}$ \\
\hline 10 & 4.71234 & 3.3289 & 1.4161 & 4.7124 & 3.3295 & 1.4167 \\
\hline 20 & 4.7123 & 3.3283 & 1.4155 & 4.7124 & 3.3289 & 1.4161 \\
\hline 30 & 4.7118 & 3.3256 & 1.4115 & 4.7124 & 3.3284 & 1.4155 \\
\hline 40 & 4.7122 & 3.3264 & 1.4128 & 4.7124 & 3.3271 & 1.4141 \\
\hline 50 & 4.7126 & 3.3275 & 1.4145 & 4.7124 & 3.3266 & 1.4143 \\
\hline 60 & 4.7125 & 3.3269 & 1.4139 & 4.7124 & 3.3262 & 1.4138 \\
\hline 70 & 4.7123 & 3.3262 & 1.4133 & 4.7124 & 3.3259 & 1.4132 \\
\hline 80 & 4.7121 & 3.3255 & 1.4127 & 4.7124 & 3.3254 & 1.4127 \\
\hline
\end{tabular}

\section{CONCLUSION}

In this study, the numerical solutions of the MEW equation with the appropriate initial and boundary conditions have been obtained by the Strang splitting techniques with help a linearized technique. To show the accurary and efficiency of the proposed numerical schemes,error norms $L_{2}$ and $L_{\alpha}$ and $I_{1}, I_{2}$ and $I_{3}$ invariants have been calculated. when error norms norms $L_{2}, L_{\alpha}$ and invariant values calculated have 
been compared with study available in the literatüre, we have find some approximate values in different time steps. It is seen that the present method produce some good results.

\section{REFERENCES}

[1] Esen,A, Kutluay,S, Solitary wave solutions of the modified equal width wave equation. Commun.Nonlinear Sci. Numer. Simul, ., 13(8), 1538-1546, 2008.

[2] Evans DJ and Raslan KR, Solitary waves for the generalized equal width (GEW) equation. Int J Comp Math, 82(4), 445-55. 2005.

[3] Geizer J, Recent advances in splitting methods for multi physics and multiscale.Theory and Applications, Journal of Algorithms and Computational Technology, 9(1), 65-93, 2013.

[4] Hamdi S, Enright WH, Schiesser WE, Gottlieb JJ, Exact solutions of the generalized equal width wave equation. ICCSA, 2, 725-34, 2003.

[5] Karakoç B, Uçar, Y, Yağmurlu NM, Different linearization tecniques for the numerical solution of the MEW equation.Selçuk Journal of Applied Mathematics, 2(13), 43-62, 2012.

[6] Karakoç and Geyikli., A numerical solution of the MEW equation using sextic B-splines.Journal of Advanced Research in Applied Mathematics, 3(5), 51-65, 2013.

[7] Raslan K. R ,A.Ramadan, M, Ameen, I.G, Finite difference approximatons for the modified equal width wave (MEW) equation. J.Math.Comput. Sci, 5,940-957, 2014.

[8] Keskin,P,Irk, D, Numerical solution of MEW equation by using finite difference method. Word Applied sciences Journal , ., , 12(2), 197-20, 2011.

[9] Marchuk G.I, Some application of splitting-up methods to the solution of mathematical physics problems, Aplikace matematiky, ,13, 103-132, 1968.

[10] Morrison PJ, Meiss JD, Carey JR , Scattering of RLW solitary waves. Physica D , 11, 324-36, 1984.

[11 ]P. J. Olver, Euler operators and conservation laws of the BBM equations, Math. Proc. Camb. Phil. Soc, 85: 143-159.

[12 ] Saka B, Algorithms for numerical solution of the modified equal width wave equation using collocation method. Mathematical and Computer Modelling, 1979, 45,1096-1117, 2007.

[13] Smith G.D, Numerical Solution of Partial Differential Equations. Finite Difference Methods, 1985, Clarendon Press, Oxford.

[14] Stanley G, Rubin and Randolph A. Graves, A , Cubic spline approximation for problems in fluid mechanics. Nasa TR R-436, Washington, DC., 1975.

[15] Strang G, On the construction and comparison of difference schemes, SIAM J. Numer. Anal, 5 (3) ,506-517, 1968.

[16] Wazwaz AM, The tanh and sine-cosine methods for a reliable treatment of the modified equal width equation and its variants. Commun Nonlinear Sci Numer Simul , 11:148-60, 2006.

[17] Varadarajan S, Lie groups, lie algebras, and their representations (First Edition), Springer-Verlag New York.

[18] Zaki SI, Solitary wave interactions for the modified equal width equation. Comput Phys Commun , 219-31, 2000. 